\title{
Corrosion Inhibition of Mild Steel in 1.0 M HCl by two Hydrazone Derivatives
}

\author{
H. Lgaz ${ }^{1}$, S. Zehra ${ }^{2}$, M. R. Albayati ${ }^{3}$, K. Toumiat ${ }^{4}$, Y. El Aoufir ${ }^{5}$, A. Chaouiki ${ }^{6}$, R. Salghi ${ }^{6 *}$, Ismat. \\ H. Ali ${ }^{7}$, M. I. Khan ${ }^{8}$, I-M. Chung ${ }^{1 *}$, S. K. Mohamed ${ }^{9}$ \\ ${ }^{1}$ Department of Crop Science, College of Sanghur Life Science, Konkuk University, Seoul 05029, \\ South Korea \\ ${ }^{2}$ Corrosion Research Laboratory, Department of Applied Chemistry, Faculty of Engineering and \\ Technology, Aligarh Muslim University, Aligarh, India \\ ${ }^{3}$ Department of Chemistry, College of Education, Kirkuk University, Kirkuk, Iraq \\ ${ }^{4}$ Department of Materials Sciences, Laghouat University, PO Box 37, 03000, Laghouat, Algeria \\ ${ }^{5}$ Materials, Nanotechnology and Environment Laboratory, Faculty of Sciences, Mohammed V \\ University Rabat, Morocco. \\ ${ }^{6}$ Laboratory of Applied Chemistry and Environment, ENSA, University Ibn Zohr, PO Box 1136, \\ Agadir, Morocco \\ ${ }^{7}$ Department of Chemistry, College of Science, King Khalid University, P. O. Box 9004, Postal Code \\ 61413, Abha, Kingdom of Saudi Arabia \\ ${ }^{8}$ Chemical Engineering Department, College of Engineering, King Khalid University, Abha, Kingdom \\ of Saudi Arabia \\ ${ }^{9}$ Faculty of Science and Engineering, Health Care Division, Manchester Metropolitan University, \\ Manchester M1 5GD, England \\ *E-mail: r.salghi@uiz.ac.ma, imcim@konkuk.ac.kr
}

doi: $10.20964 / 2019.07 .08$

Received: 7 January 2019 / Accepted: 14 April 2019 / Published: 10 June 2019

In this paper, a combined experimental and theoretical study was conducted to explain the scientific mechanism of adsorption of two hydrazone derivatives (HDZs) containing mefenamic acid (MA) namely, (E)-2-((2,3-dimethylphenyl)amino)-N'-(thiophen-2-ylmethylene)benzohydrazide (HDZ-S) and (E)-2-((2,3-dimethylphenyl)amino)-N'-(furan-2-ylmethylene)benzohydrazide (HDZ-O) on mild steel (MS) in $1.0 \mathrm{M} \mathrm{HCl}$. Electrochemical techniques and scanning electron microscope (SEM) were performed to evaluate the corrosion inhibition performances. Electrochemical results disclosed that the two compounds could effectively control the dissolution rate of mild steel in acidic medium through physicochemical adsorption following Langmuir adsorption model. Potentiodynamic polarization curves indicated that the furocoumarin molecules could be classified as mixed-type inhibitors by preventing anodic metal dissolution and cathodic hydrogen evolution reaction. The results of SEM experiment agree with electrochemical results and confirm the effective adsorption of both compounds on the steel surface. 
Keywords: Corrosion inhibition; Mild steel; Hydrazone derivative; HCl; SEM.

\section{$\underline{\text { FULL TEXT }}$}

(C) 2019 The Authors. Published by ESG (www.electrochemsci.org). This article is an open access article distributed under the terms and conditions of the Creative Commons Attribution license (http://creativecommons.org/licenses/by/4.0/). 